\section{Follow-up at One Year}

A further postal follow-up was carried out after one year. Only 262 $(57 \%)$ of the original entrants to the trial returned their forms, and $66 \%$ of those who replied said that they were still having backache. The highest proportion (79\%) was in the physiotherapy group but the differences between treatments were not significant. This backache was not equally severe in all patients, and $49 \%$ reported that no attack had prevented them from working in the year since treatment. Twenty-one per cent. reported continuous pain which prevented them from working, and the others reported varying numbers of episodes. There were no important differences between treatments though the analgesic group had rather more patients with continuous pain and fewer with acute attacks than the other groups. Of 151 patients who had more pain $62 \%$ reported episodes lasting less than a week, $11 \%$ reported episodes of one to four weeks, and $27 \%$ reported episodes lasting over a month. There were no significan differences among the treatment groups.

Finally, those who had had more treatment during the year were asked to indicate which of the following were most helpful: none, tablets, traction, corset, manipulation, bed rest, heat, exercises, or any other treatment (to be specified). The replies covered nearly every possible combination. A quarter found tablets alone most helpful (to prevent confusion we had not asked which had been used). Twelve patients thought manipulation most helpful and another 12 found the corset (with or without tablets) best, but no other combination was mentioned by more than two patients. One patient originally treated by manipulation finally resorted to acupuncture.

\section{Conclusion}

Clearly, none of the methods of treating low back pain compared in this trial showed any great superiority. Patients treated with analgesics alone fared marginally worse than those on the other three treatments. In the long term the corset was as effective as the other treatments, and it is certainly less expensive than manipulation or physiotherapy and safer than drugs. Manipulation produced an early response in a few cases, but our results suggest that there is little point in continuing to manipulate patients who show no early improvement. Nothing found at the initial assessment enabled us to identify in advance the relatively small number of patients who benefited from manipulation.

We thank the original planning committee of the trial: $\mathrm{Dr}$ R. C. B. Barbor, Dr. D. A. Brewerton, Dr. M. E. B. Carson, and Mr. R. Campbell Connolly; Mr. J. S. Batohelor, Mr. M. H. M. Harrison, and Mr. C. C. Jeffrey, who represented the British Orthopaedic Association; and Mr. G. P. Grieve, representing the Chartered Society of Physiotherapy, who acted as honorary secretary to the working party.

Special thanks are due to all our colleagues whose departments took part in the trial: Dr. F. S. Cooksey and Dr. E. B. D. Hamilton at King's College Hospital, Dr. K. N. Lloyd at University Hospital of Wales, Cardiff, Dr. H. Rhys Davies at Peace Memorial Hospital, Watford, Dr. A. Stoddard at Brook General Hospital, Dr. D. A. H. Yates at St. Thomas's Hospital, and Dr. A. Zinovieff at Dryburn Hospital, Durham.

Miss Margaret Black, secretary to the department of rheumatology at the West Middlesex Hospital, Isleworth, played a major part in co-ordinating the documentation and in organizing the whole follow-up. We also thank Mrs. Stella Lyons of the department of medical statistics, Mrs. Jessie Rogers of the medical care research unit, and Dr. Nona Newman of the computer department, University of Newcastle upon Tyne. Dr. J. B. Spooner of the Sterling-Winthrop Group kindly arranged for the supply of all the analgesic tablets used in the trial and these were provided gratis by the makers.

\section{Reference}

${ }^{1}$ British Association of Physical Medicine, British Medical fournal, 1961, 1, 253.

\title{
Hydroxyproline Excretion in Patients with Breast Cancer and Response to Treatment
}

\author{
T. J. POWLES, C. L. LEESE, P. K. BONDY
}

British Medical fournal, 1975, 2, 164-166

\section{Summary}

The urinary excretion of hydroxyproline, measured as the hydroxyproline:creatinine ratio, was useful in monitoring the progression of metastatic cancer of the breast. After new treatment was started changes in the hydroxyproline excretion occurred earlier than other clinically observable responses. The test could therefore be used for predicting the response to treatment and early detection of the sensitivity of the tumour to hormone therapy.

\section{Introduction}

Hydroxyproline excretion is reported to be a sensitive index of bone metastases. ${ }^{12}$ Most urinary hydroxyproline originates

\footnotetext{
Department of Medicine, Royal Marsden Hospital, Sutton, Surrey SM2 5PT

T. J. POWLES, M.B., M.R.C.P., Senior Lecturer and Honorary Consultant

C. L. LEESE, PH.D., Senior Lecturer in Biochemistry

P. K. BONDY, M.D., F.R.C.P., Cancer Research Campaign Professor of Medicine
}

from bone, ${ }^{3}$ mainly from degradation of newly synthesized collagen. ${ }^{45}$

Breast tumours break down bone in vitro by release of osteolytic substances, ${ }^{6}$ and development of tumour deposits in bone may depend on this property, which may be reflected in hydroxyproline excretion. Therefore, successful treatment of metastatic breast cancer may be associated with a decrease in hydroxyproline owing to a decrease in bone destruction, whereas progression of tumour growth with unsuccessful treatment may be associated with increased hydroxyproline excretion. To examine this hypothesis we estimated hydroxyproline excretion in patients with metastatic breast cancer before, during, and after various treatments.

\section{Patients and Methods}

Thirty-one patients with metastatic breast carcinoma were admitted to the metabolic ward for full metabolic and biochemical assessment. This included thorough clinical evaluation; bone scan (Tc-polyphosphate); liver scan by isotopic methods and B-mode ultrasound; chest $x$-ray examination; modified skeletal survey; marrow aspiration; urinary and serum calcium, phosphorous, and magnesium estimation; and liver function tests. Evaluation of the patients' progress was made jointly by surgeon, physician, and radiotherapist and recorded in the patients' notes. Urinary hydroxyproline excretion was not estimated 
on admission but stored for later estimation, so clinical assessment of the patient was independent of these results.

Patients were treated with quadruple chemotherapy (cyclophosphamide, fluorouracil, methotrexate, and vincristine), hormone therapy (ethinyloestradiol $0.3 \mathrm{mg} /$ day or nandrolone phenylpropionate $100 \mathrm{mg} /$ week), corticosteroid therapy (prednisolone $7 \cdot 5-20 \mathrm{mg} /$ day), endocrine ablation (oophorectomy), or combinations of these. Several patients were studied sequentially on more than one type of treatment and some patients were studied after stopping ineffective therapy and before starting new therapy. Altogether 41 treatment (or non-treatment) episodes were studied. Objective remission was considered to have occurred if there was a reduction of at least $50 \%$ in tumour volume or an independent radiological report of regression and recalcification of lytic bone metastases.

Total hydroxyproline in the urine was estimated by the method of Prockop and Udenfriend, ${ }^{7}$ in which hydroxyproline in the urine is oxidized to pyrrole extracted with toluene and estimated by colour reaction with Erlich's reagent. The method measures both free and peptide-bound hydroxyproline. Results were expressed as either total hydroxyproline excretion or hydroxyproline excretion per unit of excreted creatinine (hydroxyproline : creatinine ratio) calculated as urinary hydroxyproline $(\mathrm{mg} /$ day $) \times 100 /$ urinary creatinine $(\mathrm{mg} /$ day $)$. The two ways of expressing hydroxyproline excretion correlated closely $(r=0.91 ; P=<0.01)$.

\section{Results}

Duplicate samples from 68 untreated patients with various stages of disease showed a mean variation $( \pm S . D$.) in hydroxyproline : creatinine excretion ratio of $0.72 \pm 0.68$ for consecutive 24-hour samples. Therefore changes in the hydroxyproline : creatinine excretion ratio in the patients being treated were considered significant if they were greater than 2.08 -that is the mean variation +2 S.D. in duplicate samples.

Of the 41 different treatment (or non-treatment) manoeu vres studied 10 were associated with definite clinical or radiological improvement or both and in all of these the hydroxyproline : creatinine ratio decreased (fig. 1). The mean decrease was $6.8+4.9 / P<0.002)$ and in nine out of the 10 the change was significant $(>2.08)$. Five of these patients had serial estimations and the decrease in excretion of hydroxyproline occurred between two and eight weeks (median 4.5 weeks) before clinical evidence of response to therapy appeared.

Clinical deterioration during treatment occurred in 20 of the 41 treatment manoeuvres and in each case the hydroxyproline : creatinine ratio increased (fig. 1). The mean increase for the 20 was $7 \cdot 0 \pm 5 \cdot 2$ $(P<0.005)$ and in 18 out of the 20 the changes were significant. Serial estimations were carried out about every three weeks in 11 patients and increases in their hydroxyproline : creatinine ratios

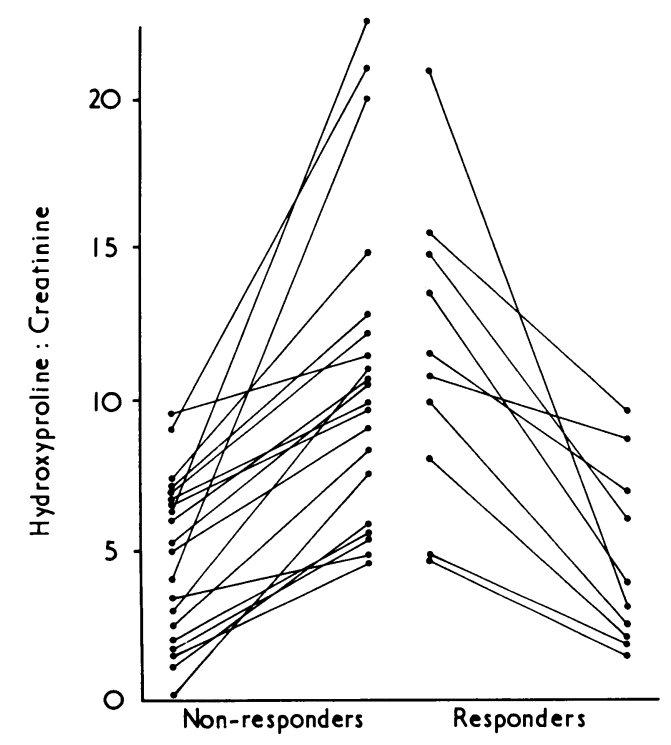

FIG. 1-Changes in hydroxyproline excretion in patients with breast cancer during 20 failed and 10 successful courses of treatment. occurred from 0 to 26 weeks (median 7.6 weeks) before clinical deterioration justified change of therapy.

Total urinary hydroxyproline excretion reflected response to treatment but not as well as the hydroxyproline : creatinine ratio. There was no correlation between creatinine excretion and response to treatment. Serial estimations of the hydroxyproline : creatinine excretion ratio were performed in two patients with metastatic breast cancer and oestrogen-induced hypercalcaemias. Significant changes occurred within a few days in parallel with changes in the serum calcium (fig. 2).

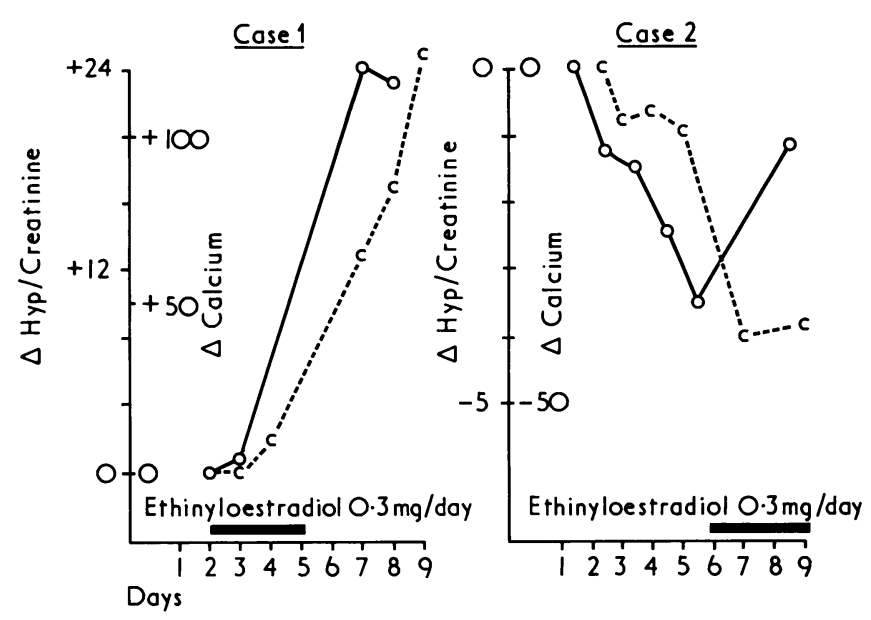

FIG. 2-Changes in urinary excretion of hydroxyproline and serum calcium during oestrogen-induced hypercalcaemia (case 1) and one week after cessation of oestrogen-induced hypercalcaemia (case 2). $\triangle \mathrm{Hyp} / \mathrm{Creatinine}=$ Change in hydroxyproline : creatinine ratio. $\triangle$ Calcium $=$ Change in serum calcium (mg/l).

\section{Discussion}

Total hydroxyproline excretion or hydroxyproline : creatinine excretion ratio decreased in patients with metastatic breast cancer who responded to treatment and increased in those who failed to respond, whatever the type of therapy. This suggests that tumour regression is associated with decreased and tumour progression with increased breakdown of collagen and that hydroxyproline excretion can be used as a monitor for response to treatment in this disease.

Though creatinine excretion showed no correlation with response to treatment the hydroxyproline : creatinine excretion ratio was more sensitive than the estimation of total hydroxyproline alone. This may represent a correction for incomplete urine collection, particularly in those patients who were unwell enough to make accurate collection difficult.

Many factors, including diet, age, sex, drugs, and hormones, may influence hydroxyproline excretion. ${ }^{1}$ Oestrogen and androgen therapy will reduce hydroxyproline excretion in persons with no malignant disease, perhaps by reduction of physiological bone resorption. ${ }^{8}$ Patients with metastatic bone disease have increased bone resorption, and therefore the antitumour effects of these hormones may influence the hydroxyproline excretion more than the physiological effects. This would account for the close relation between the hydroxyproline excretion and response to therapy with these hormones seen in our patients. Corticosteroid therapy does not influence hydroxyproline excretion in patients without cancer. ${ }^{9}$

Changes in hydroxyproline excretion occurred in many patients several weeks before apparent clinical change. Most patients were not tested early or often enough during the initial phase of treatment to determine the prediction value of the test, though two patients who were tested daily showed very rapid changes. Eleven patients who had serial estimations about every three weeks had a mean delay of 7.6 weeks between increase in 
hydroxyproline excretion and change of treatment on clinical grounds.

In the two hypercalcaemic patients hydroxyproline excretion changed rapidly in parallel with changes in serum calcium. This suggests that the test was monitoring changes in bone destruction in relation to tumour treatment and encourages the hypothesis that estimation of hydroxyproline excretion in the first few days of therapy may be usefun for early detection of hormone-sensitive tumours and early prediction of response to therapy.

We thank Dr. J. W. Baker Dr. H. T. Ford Mr. J. C. Gazet Mr. A. McKinna, and Mr. A. York Mason for their very close clinical cooperation with the department of medicine which made it possible for these patients to be studied.
This study was supported by the Ludwig Institute of Cancer Research and the Medical Research Council.

\author{
References \\ ${ }^{1}$ Cuschieri, A., British fournal of Surgery, 1973, 60, 800 \\ Sumner, D. S., et al., British fournal of Surgery, 1973, 60, 306 \\ Dull, T. A., and Henneman, P. H., New England fournal of Medicine, \\ $1963,268,132$ \\ ${ }^{4}$ Lindstedt, S., and Prockop, D. J., fournal of Biological Chemistry, 1961, \\ 236, 1399.
5 Prockop, D. Journal of Clinical Investigation, 1964, $43,453$. \\ 6 Powles, T. J., et al., British fournal of Cancer, 1973, 28, 316. \\ Prockop, D. J., and Udenfriend, S. A., Analytical Biochemistry, 1960, 1, \\ 228. \\ Katz, F. H., and Kappas, A., fournal of Laboratory and Clinical Medicine, \\ $1968,71,65$. \\ ${ }^{9}$ Ziff, M., et al., Fournal of Clinical Investigation, 1956, 35, 579.
}

\title{
Occult Pulmonary Haemorrhage in Leukaemia
}

\author{
DAVID W. GOLDE, \\ MARTIN J. CLINE

\section{W. LAWRENCE DREW， HARVEY Z. KLEIN， THEODORE N. FINLEY,}

British Medical fournal, 1975, 2, 166-168

\section{Summary}

Though bleeding into the lung parenchyma is responsible for morbidity and mortality in patients with leukaemia pulmonary haemorrhage is seldom diagnosed during life. We diagnosed occult pulmonary haemorrhage in five leukaemic patients with unexplained infiltrates on chest roentgenograms by examining alveolar macrophages retrieved by bronchopulmonary lavage. Macrophage haemosiderin content was greatly increased in the patients with pulmonary haemorrhage as compared to normal and thrombocytopenic control subjects. Haemoglobin and intact erythrocytes in alveolar macrophages were taken as evidence of recent haemorrhage. Intrapulmonary bleeding may occur often in patients with leukaemia, and bronchopulmonary lavage offers a safe approach to diagnosis and allows for concomitant identification of pulmonary infection.

\section{Introduction}

In patients with leukaemia pulmonary infiltrates visible on chest roentgenograms are often difficult to evaluate. Infection, particularly by "opportunistic pathogens," is a common cause of pulmonary disease in leukaemic patients, ${ }^{1-3}$ and leukaemic infiltration of the lung parenchyma is often considered in making a diagnosis. ${ }^{2}$ Pulmonary haemorrhage associated with thrombocytopenia, however, is not often diagnosed clinically and the possibility of uncontrolled bleeding makes invasive diagnostic procedures such as lung biopsy and bronchial brushing hazardous. ${ }^{4} 5$

\footnotetext{
Division of Haematology and Oncology, U.C.L.A. School of Medicine, Los Angeles, California, U.S.A.

DAVID W. GOLDE, M.D., F.A.C.P., Assistant Professor of Medicine

MARTIN J. CLINE, M.D., F.A.C.P., Bowyer Professor of Medical Oncology Mount Zion Hospital, San Francisco, California, U.S.A. W. LAWRENCE DREW, M.D., PH.D., Director of Clinical Microbiology HARVEY Z. KLEIN, M.D., Director of Tissue Pathology THEODORE N. FINLEY, M.D., Professor of Medicine
}

We describe here five leukaemic patients with unexplained pulmonary infiltrates on their chest films in whom intrapulmonary haemorrhage was diagnosed.

\section{Patients}

Case 1.-A 12-year-old black boy with acute myelogenous leukaemia presented with anaemia, bleeding of the gums, and profound thrombocytopenia. He was treated with a combination of cyclophosphamide, vincristine, cytarabine, and prednisone (COAP), ${ }^{6}$ then daunorubicin. A partial remission was achieved and he remained at home until he was readmitted for a nonproductive cough and fever. A chest roentgenogram showed a right basilar infiltrate. Haemoglobin was $5.5 \mathrm{~g} / \mathrm{dl}$ and the platelets $5 \times 10^{\circ} / 1$. A sputum culture grew normal oral flora. He was given red-cell and platelet transfusions and treated with a combination of cephalothin, gentamicin, and cleomycin. The chest film showed gradual clearing of the right lower lobe process over the next few days. On the seventh day in hospital, however, new infiltrates appeared in both upper lobes. Pulmonary lavage was performed. The lavage fluid was grossly bloody and no infectious agent was identified on direct smear or by culture. The pulmonary infiltrates subsequently cleared without further antibiotic treatment. One month later the patient died with diffuse bleeding and fulminant leukaemia.

Case 2.-A 54-year-old man with acute myelogenous leukaemia was initially treated with COAP and then with cytarabine, thioguanine, and daunorubicin. His remission was short-lived. He developed fever and petechial haemorrhages of the skin, and diffuse nodular infiltrates were seen on a chest film. The platelet count was $14 \times 10^{2} / 1$. There was no haemoptysis. Pulmonary lavage was performed and a nonpathogenic fungus, thought to be a contaminant, was cultured from the blood-tinged lavage fluid.

Case 3.-A 50-year-old woman with acute myelogenous leukaemia achieved a remission with COAP therapy. The disease subsequently relapsed and was treated with thioguanine. During treatment the patient became febrile and was severely leucopenic and thrombocytopenic. Diffuse bilateral nodular infiltrates were seen on a chest roentgenogram and pulmonary lavage was performed. The lavage fluid was pink and sterile on culture. The patient subsequently died with uncontrolled leukaemia, bleeding, and staphylococcal septicaemia.

Case 4.-A 12-year-old Latin American boy with acute myelogenous leukaemia was treated initially with COAP, then daunorubicin, and later cytarabine and thioguanine. A remission was not achieved. Pulmonary lavage was performed to evaluate a persistent pulmonary infiltrate. At this time the patient had uncontrolled leukaemia with a peripheral white count of $176 \times 10^{\circ} / 1$. Bacillus cereus was cultured from the bloody lavage fluid, but this was thought to be a contaminant. The patient was maintained on antibiotics and treated with platelet 